\title{
Software News and Update pyVib, A Computer Program for the Analysis of Infrared and Raman Optical Activity
}

\author{
MOHAMED ZERARA \\ Institut de chimie physique, Université de Fribourg, 9, Chemin du musée, CH-1700 Fribourg, \\ Switzerland
}

\begin{abstract}
A new program called pyVib has been developed as a tool for the analysis of Gaussian (Gaussian 03, Gaussian Inc., Pittsburgh, PA) outputs of vibrational absorption (IR), Raman as well as vibrational optical activity (VOA) spectra calculations. This program has been designed to help the computational chemistry practitioner in the task of analyzing and visualizing molecular vibrations and cross sections. In particular, the analysis of absorption and scattering cross sections can be done using new tools such as group coupling matrices (GCMs) and atomic contribution patterns (ACPs) as either 2D or 3D representations, respectively (Hug, Chem Phys 2001, 264, 53). It reads the Hessian, the atomic polar tensors (APTs), the atomic axial tensors (AATs) (Nafie, J Chem Phys 1983, 79, 4950), and the gradients of the various polarizability tensors involved in VOA calculations and stored in Gaussian $f c h k$ ascii files. pyVib is capable of picking suitably chosen atoms or group of atoms for evaluating the contribution of each atom or defined groups of atoms to the calculated VOA scattered intensities. All the results generated by pyVib can be visualized in real-time but can also be transferred to text editors and electronic spreadsheets, which facilitate a detailed subsequent analysis and the visualization by other graphical user interfaces (GUIs).

This program is coded in Python and used the visualization toolkit (VTK) library. It is freely available under the terms of the general GNU public license (GPL) for Linux platforms*.
\end{abstract}

Key words: vibrational optical activity; group coupling matrix; atomic contribution pattern; Python; VTK

\section{Introduction}

Visualization is a convenient and powerful way to interpret the vast amount of data generated by some daily computational chemistry tasks. Therefore, there might be a need for efficient programs for analyzing and visualizing large data sets generated by vibrational absorption, Raman scattering, and vibrational optical activity calculations. To address these needs, we have developed a new program, which combines a graphical user interface and data-analysis routines.

Among other capabilities, the molecular graphics program pyVib has been intended to visualize molecular structures and vibrations. pyVib is able to read both the optimized geometry and the Hessian from the fchk ascii text files generated by Gaussian ab initio quantum chemical program ${ }^{1}$ and perform geometrical reconstructions of molecular structures and vibrational analysis. Additionally, it reads the gradients of electric dipole-electric dipole, electric dipole-electric quadrupole, and electric dipole-magnetic dipole polarizabilities and calculates two Raman invariants, $a^{2}$ and $\beta^{2}$, and three vibrational Raman optical activity (ROA) invariants, $a G^{\prime}$,
$\beta_{G}^{2}$, and $\beta_{A}^{2}$ for each vibrational mode of the molecule. ${ }^{2}$ Similarly, pyVib reads the atomic polar and axial tensors, APTs and AATs, respectively, to calculate vibrational absorption (IR) and vibrational circular dichroism (VCD) invariants ${ }^{3}$ and, in turn, cross sections. Figure 1 summarizes the capabilities of pyVib.

To date, although some routines, ${ }^{\dagger}$ which necessite the Matlab proprietary software, have been developed by the group of Hug (University of Fribourg, Switzerland), pyVib is the only freely available standalone software, which can visualize the atomic contribution patterns (ACPs) and group coupling matrices (GCMs) as defined by Hug. ${ }^{2}$

\section{Correspondence to: Dr. M. Zerara; e-mail: zerara@molcad.de}

Contract/grant sponsor: Swiss National Science Foundation; contract/grant numbers: 2000-066679 and 200020-103750

* pyVib can be downloaded from www.sourceforge.net/projects/pyvib

${ }^{\dagger}$ The Matlab routines can be obtained from the author 


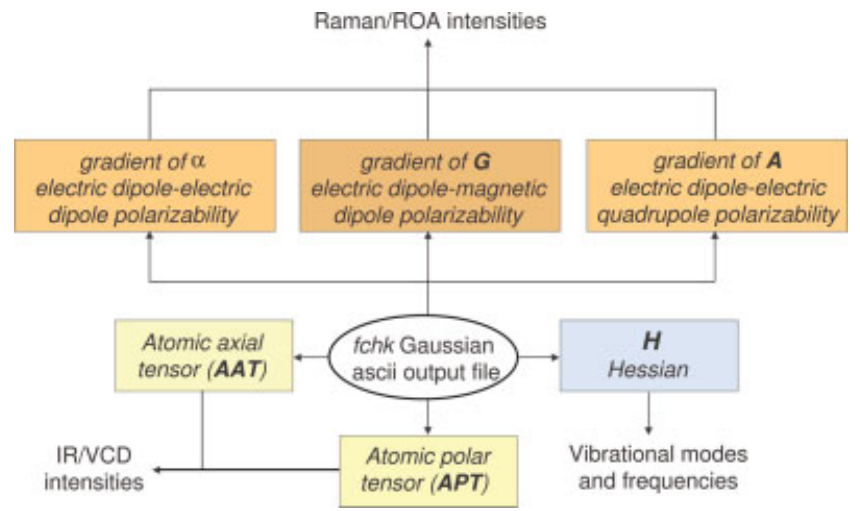

Figure 1. Main capabilities of pyVib program. It needs a fchk ascii output file to perform a vibrational analysis and IR/VCD as well as Raman/ROA cross sections analysis. [Color figure can be viewed in the online issue, which is available at www.interscience.wiley.com.]

\section{Discussion}

The program takes as input the optimized geometry, the hessian of a frequency calculation and the APTs and AATs needed for IR and VCD as well as the gradients of polarizabilities one needs for Raman and vibrational ROA type calculations. pyVib has a main interface, which allows one to select one of these four different vibrational spectroscopies, for which vibrational analysis is performed from the input Hessian, and the resulting modes can therefore be represented graphically.

\section{Representation of Vibrations and Vibrational Energies}

pyVib can represent the vibrations in two different ways. The first one consists in the classical representation of arrows where the direction of the vibration of a given nuclei correponds to the direction of the arrow while its amplitude is proportional to the length of the arrow. The second one uses spheres instead of arrows as shown in Figure 2. In this case, we exploit the three-dimensional representation of spheres centered at the equilibrium position of the nuclei involved in a given vibrational mode. The size of the spheres is taken proportional to the amplitude of the nuclear motion, and the direction of the nuclear displacement is given by the normal to the intersection plane of the two halve spheres.

To account for the importance of nuclear motions have in vibrational absorption and Raman and VOA cross sections, pyVib allows representing spheres with either surface or volume proportional to the square of the Cartesian vector displacements $L_{\alpha, p}^{x}$ (where $\alpha$ and $p$ are respectively the nuclei and the vibrational mode). As the invariants are proportional to $\left(L_{\alpha, p}^{x}\right)^{2} / \Delta v_{p}$ and, in turn, cross sections, the size of the spheres is further divided by the wave number $\Delta v_{p}$ associated to vibrational mode $p$ (except for VCD). ${ }^{4}$ This option based on the sphere representation is implemented in pyVib.

A second approach based on representation by spheres resides in considering the energy of a nuclei $\alpha, E_{\alpha, p}$, with respect to the total energy of a vibrational mode $p, E_{p} .{ }^{4}$ Starting from the virial theorem and the harmonic approximation, the fractional energy can be written as

$$
\frac{E_{\alpha, p}}{E_{p}}=m_{\alpha} L_{\alpha, p}^{x} L_{\alpha, p}^{x} .
$$

Therefore, it would be worth to set either the surface or the volume proportional to $m_{\alpha} L_{\alpha, p}^{x} L_{\alpha, p}^{x}$. Like in Gaussian, the sum of Cartesian displacements vectors is normalized so that

$$
\sum_{\alpha} L_{\alpha, p}^{x} L_{\alpha, p}^{x}=1
$$

The advantage of this condition is mostly of practical nature; the scale hardly ever needs to be readjusted when going from vibration to another. For a given vibration, but not between vibrations, the relative size of the spheres shows the relative importance nuclear motion has for generating a particular cross section. Unfortunately, comparison of the size of nuclear motion is not possible for different modes. Although, the surface and volume are, by default, chosen proportional to the displacements and kinetic energy, respectively, it is, however, also possible to chose either surface or volume for both options (excursion and kinetic).

\section{Vibrational Analysis}

The vibrational analysis performed by pyVib is not different from that described by Wilson et al. ${ }^{5}$ Frequencies and normal modes are calculated in pyVib, starting with Hessian read from the Gaussian fchk output file. The vibrational analysis follows these steps:

1. First, pyVib mass weights the $3 N \times 3 N$ Hessian and diagonalizes it to yield $3 N$ eigenvectors and $3 N$ eigenvalues.

2. Second, pyVib translates the center of mass to the origin. Then, it calculates the vectors corresponding to rotations and translations from the transformation matrix, which diagonalizes the moment of inertia tensor, leaving $3 N-6$ or $3 N-5$ vibrational modes for further analysis.

3. Third, it transforms the mass weighted Hessian to internal coordinates and diagonalizes it.

4. At this point, it converts the frequencies, which correspond to vibrational modes in units of reciprocal centimers.

5. Finally, it calculates the reduced mass, force constants, and Cartesian displacements.

\section{GCMs and ACPs}

The decomposition of Raman and ROA invariants, and dipole and rotational strengths, into mono- and di-nuclear terms leads to what Hug called GCMs, which give insight into individual contributions of particular atoms or group of atoms to the overall absorption and scattered intensity. $2,4,6$

All reduced Raman and ROA invariants, and reduced dipole and rotational strengths, noticed $J$, which do not include integration over vibrational wavefunctions, can be decomposed into mono- or di-atomic terms according to

$$
J_{p}=\sum J_{\alpha \beta, p}
$$


The corresponding invariants $I$ are then obtained as follows:

$$
I=F J_{p}
$$

with $F=\left\langle 1\left|Q_{p}\right| 0\right\rangle^{2}=\hbar /\left(400 \pi c \Delta v_{p}\right)$ for Raman, ROA invariants, and dipole strength (IR). While $F=\operatorname{Im}\left\{\left\langle 1\left|Q_{p}\right| 0\right\rangle\left\langle 1\left|P_{p}\right| 0\right\rangle\right\}=\hbar / 2$ for rotational strength (VCD). $Q_{p}$ and $P_{p}$ are vibrational coordinate and moment operators. Subscripts $\alpha$ and $\beta$ are the atoms, $p$ is the vibrational normal mode, $\hbar$ is the reduced Planck's constant, $c$ is the speed of the light, and $\Delta v_{p}$ is the Raman shift.

One has for the explicit form of $J_{\alpha \beta, p}$ :

$$
J_{\alpha \beta, p}=L_{\alpha, p}^{x} V_{\alpha \beta} L_{\beta, p}^{x}=V_{\alpha \beta}: L_{\alpha, p}^{x} L_{\beta, p}^{x}
$$

where $L_{\alpha, p}^{x}$ and $L_{\beta, p}^{x}$ are cartesian displacement vectors and $V_{\alpha \beta}$ is a dyadic characteristic of the particular invariant one considers. The elements of these dyadics have the form: ${ }^{2,4}$

$$
\begin{aligned}
& V\left(a^{2}\right)_{\alpha i, \beta j}=\frac{1}{9} \sum_{\mu, v}\left(\frac{\partial \alpha_{\mu \mu}}{\partial x_{i}^{\alpha}}\right)_{0}\left(\frac{\partial \alpha_{\nu v}}{\partial x_{j}^{\beta}}\right)_{0} \\
& V\left(\beta^{2}\right)_{\alpha i, \beta j}=\frac{1}{2} \sum_{\mu, \nu}\left[3\left(\frac{\partial \alpha_{\mu \nu}}{\partial x_{i}^{\alpha}}\right)_{0}\left(\frac{\partial \alpha_{\mu \nu}}{\partial x_{j}^{\beta}}\right)_{0}\right. \\
& \left.-\left(\frac{\partial \alpha_{\mu \mu}}{\partial x_{i}^{\alpha}}\right)_{0}\left(\frac{\partial \alpha_{\nu v}}{\partial x_{j}^{\beta}}\right)_{0}\right] \\
& V\left(a G^{\prime}\right)_{\alpha i, \beta j}=\frac{1}{9} \sum_{\mu, \nu}\left(\frac{\partial \alpha_{\mu \mu}}{\partial x_{i}^{\alpha}}\right)_{0}\left(\frac{\partial G_{v v}^{\prime}}{\partial x_{j}^{\beta}}\right)_{0} \\
& V\left(\beta_{G}^{2}\right)_{\alpha i, \beta j}=\frac{1}{2} \sum_{\mu, \nu}\left[3\left(\frac{\partial \alpha_{\mu \nu}}{\partial x_{i}^{\alpha}}\right)_{0}\left(\frac{\partial G_{\mu \nu}^{\prime}}{\partial x_{j}^{\beta}}\right)_{0}\right. \\
& \left.-\left(\frac{\partial \alpha_{\mu \mu}}{\partial x_{i}^{\alpha}}\right)_{0}\left(\frac{\partial G_{v v}^{\prime}}{\partial x_{j}^{\beta}}\right)_{0}\right] \\
& V\left(\beta_{A}^{2}\right)_{\alpha i, \beta j}=\frac{\omega_{0}}{2} \sum_{\mu, \nu}\left(\frac{\partial \alpha_{\mu \nu}}{\partial x_{i}^{\alpha}}\right)_{0}\left(\frac{\partial \mathcal{A}_{\mu \nu}}{\partial x_{j}^{\beta}}\right)_{0} \\
& V(D)_{\alpha i, \beta j}=\operatorname{Re}\left\{\sum_{\mu}\left(\frac{\partial \mu}{\partial x_{i}^{\alpha}}\right)_{0}\left(\frac{\partial \mu_{\mu}}{\partial x_{j}^{\beta}}\right)_{0}\right\}=\operatorname{Re}\left\{\sum_{\mu} P_{i \mu}^{\alpha} P_{j \mu}^{\beta}\right\} \\
& V(R)_{\alpha i, \beta j}=\operatorname{Re}\left\{\sum_{\mu}\left(\frac{\partial \overrightarrow{\mu_{\mu}}}{\partial x_{i}^{\alpha}}\right)_{0}\left(\frac{\partial \overrightarrow{m_{\mu}}}{\partial \dot{x}_{j}^{\beta}}\right)_{0}\right\}=\operatorname{Re}\left\{\sum_{\mu} P_{i \mu}^{\alpha} M_{j \mu}^{\beta}\right\}
\end{aligned}
$$

The indices $i$ and $j$ refer to the Cartesian coordinates of the nuclei $\alpha$ and $\beta$, and the indices $\mu$ and $v$ to the Cartesian coordinate system in which the molecular tensors are defined. The rank-two tensor $\mathcal{A}$ is obtained by contracting the rank-three electric dipole-electric quadrupole tensor $A$ with the antisymmetric unit tensor of LeviCivita. $\vec{\mu}$ stands for the electric and $\vec{m}$ for the magnetic dipole moment, and $P_{i \mu}^{\alpha}$ and $M_{j \mu}^{\beta}$ are the components of APTs and AATs, respectively.
The sign and the size of the contribution of each atom to the intensity is revealed by the sums of elements over its corresponding row and column, according to

$$
J_{\alpha, p}=\sum_{\beta} J_{\alpha \beta, p}
$$

Unfortunately, for medium and large molecules, a numerical matrix and even, a graphical representation of the scalar components of this matrix, will be too detailed a description for allowing intuitive grasp on the size and sign of scattering for a particular mode. Therefore, it might be preferable to lump together contributions from individual atoms into chemical groups (e.g., $\mathrm{CH}_{3}, \mathrm{CH}_{2}, \mathrm{COOH}$, etc). This approach leads to GCMs, which have extensively been described in literature. ${ }^{2}$ GCMs are obtained by adding up intragroup (group of atoms) mono- and di-nuclear terms, and by representing them graphically. The contributions of defined groups are represented as circles in two-dimensional upper triangular matrices. To account for the nonsymmetry of GCM matrices corresponding to invariants $a G^{\prime}, \beta_{G}^{2}$, and $\beta_{A}^{2}$, all off-diagonal GCM matrix elements are the sums of cross terms $J_{\alpha \beta, p}+J_{\beta \alpha, p}$. The surface area of the circles is chosen proportional to the size of the molecular properties that are scalars; signs being indicated by dark and light shadings, respectively (Figs. 3 and 4).

ACPs that are derived from GCMs by further splitting of dinuclear terms would appear preferable when quantities attributable to individual atoms are needed. To this end, an allocation of fractions of dinuclear terms, through using weighting coefficients ${ }^{7}$ is done:

$$
J(\alpha)_{p}=\sum_{\beta}\left[J_{\alpha \beta, p} r(\alpha)_{\alpha \beta, p}+J_{\beta \alpha, p} r(\alpha)_{\beta \alpha, p}\right]
$$

The weighting coefficients $r(\alpha)_{\alpha \beta, p}$ and $r(\alpha)_{\beta \alpha, p}$ are calculated using the following expressions

$$
r(\alpha)_{\alpha \beta, p}=\frac{\left|L_{\alpha, p}^{x} V_{\alpha \beta}\right|+\left|L_{\alpha, p}^{x} V_{\alpha \alpha}\right|}{\left|L_{\alpha, p}^{x} V_{\alpha \beta}\right|+\left|L_{\alpha, p}^{x} V_{\alpha \alpha}\right|+\left|V_{\alpha \beta} L_{\beta, p}^{x}\right|+\left|V_{\beta \beta} L_{\beta, p}^{x}\right|}
$$

and

$$
r(\alpha)_{\beta \alpha, p}=\frac{\left|V_{\beta \alpha} L_{\alpha, p}^{x}\right|+\left|V_{\alpha \alpha} L_{\alpha, p}^{x}\right|}{\left|V_{\beta \alpha} L_{\alpha, p}^{x}\right|+\left|V_{\alpha \alpha} L_{\alpha, p}^{x}\right|+\left|L_{\beta, p}^{x} V_{\beta \alpha}\right|+\left|L_{\beta, p}^{x} V_{\beta \beta}\right|}
$$

With cross sections representing areas, and considering the fact that they represent quantities for an isotropic sample and thus rotational averages, the logical choice is to draw spheres with a surface proportional to the computed cross sections (Fig. 4).

\section{Cross Sections}

The theoretical expressions for circular difference scattering $(\mathrm{ROA})^{8}$ and circular difference absorption (VCD) ${ }^{9}$ are well established for isotropic samples such as liquids (both methods) as well as 

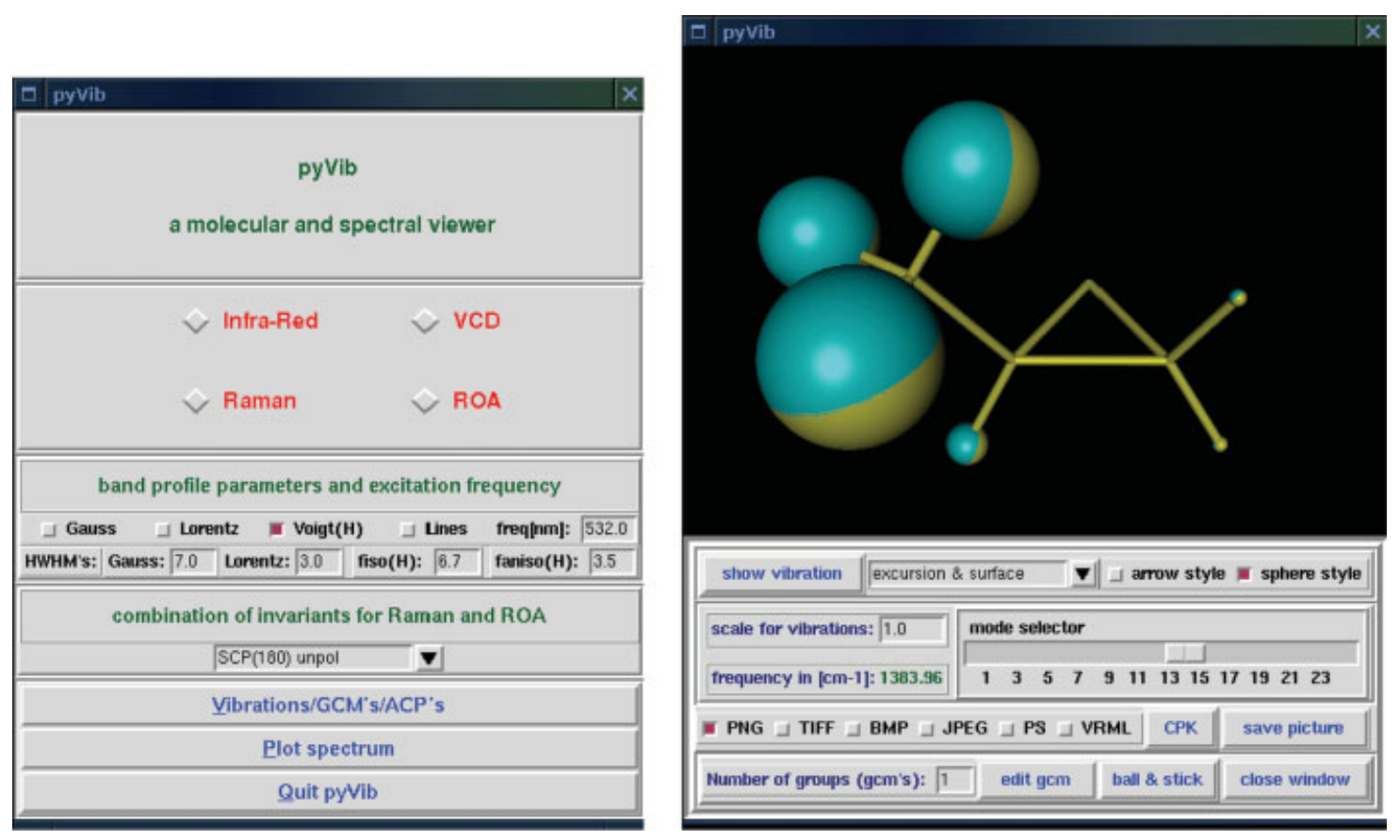

Figure 2. Left: The main interface of pyVib consists of one window from which it is possible to choose between different vibrational spectroscopies. Then one might select one of the two kinds of tasks, which can be performed; calculation of the spectra or GCP and ACP analysis. Right: Sphere representation used for the symmetric deformation (umbrella) mode in methyloxirane molecule. [Color figure can be viewed in the online issue, which is available at www.interscience.wiley.com.]

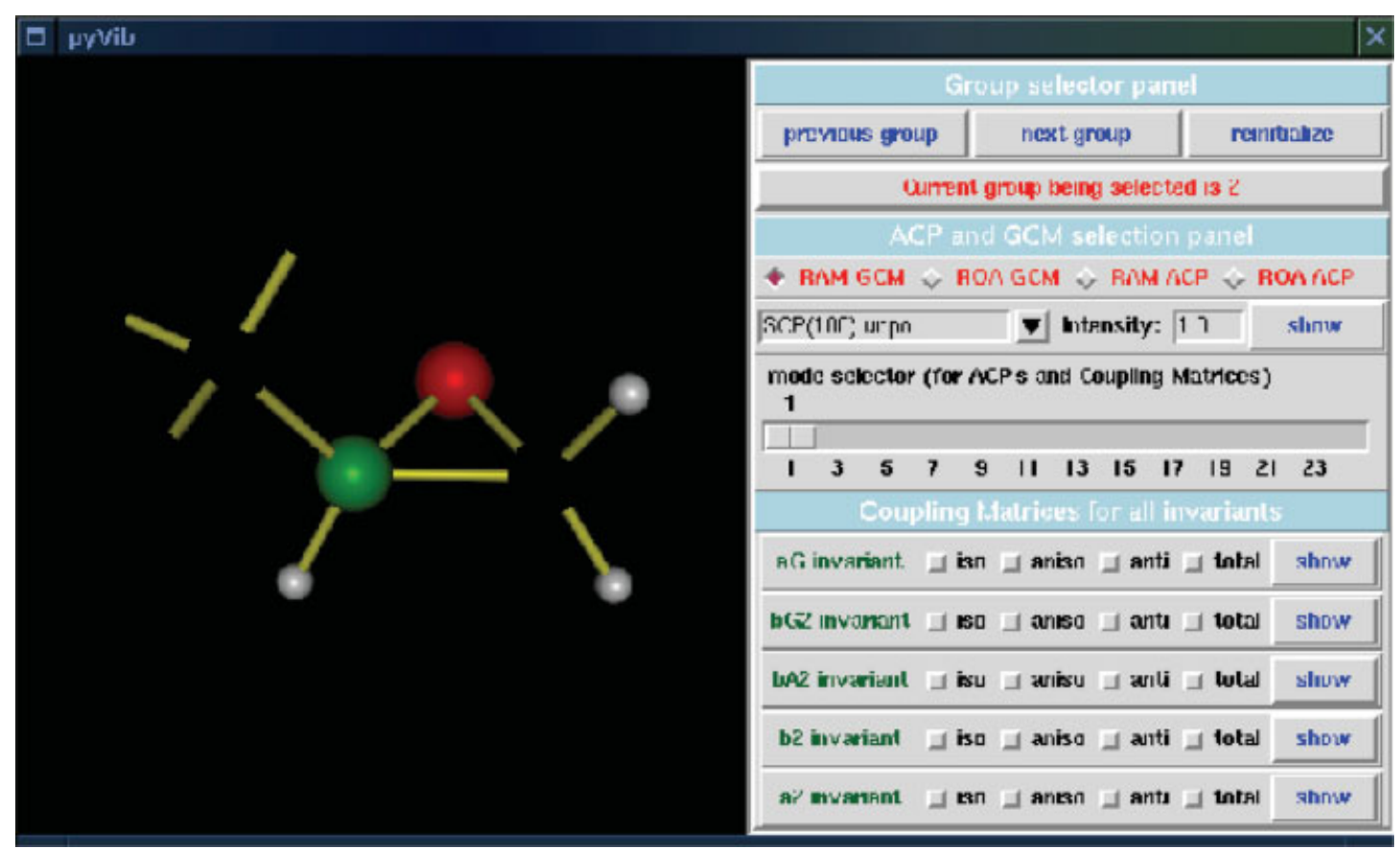

Figure 3. Interface dedicated to group selection and definition process. Then GCMs and ACPs can be visualized for different Raman/ROA and IR/VCD methods. [Color figure can be viewed in the online issue, which is available at www.interscience.wiley.com.] 

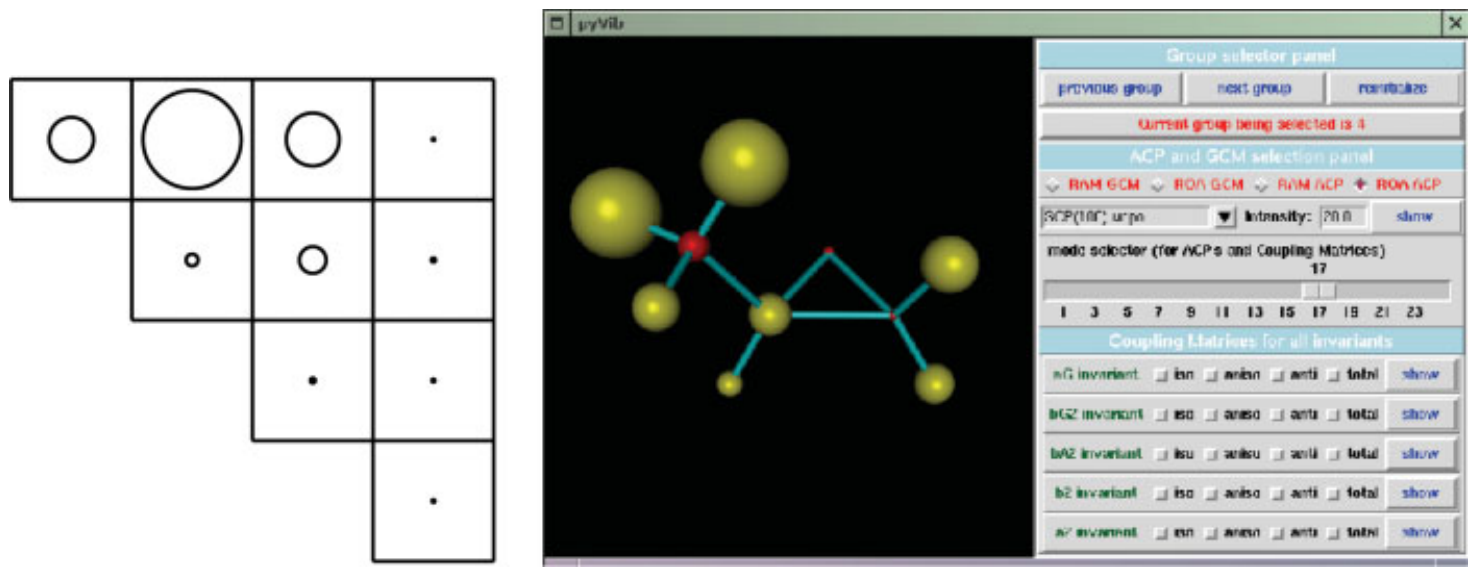

Figure 4. ROA GCM (left) and ROA ACP for mode 17 of methyloxirane (antisymmetric deformation of methyl). In this example, groups are $\mathrm{CH}_{3}, \mathrm{CH}_{2}, \mathrm{CH}$, and $\mathrm{O}$. [Color figure can be viewed in the online issue, which is available at www.interscience.wiley.com.]

randomly oriented crystallites embedded in a viscous solvent (only VCD to date). In vibrational absorption, intensities are commonly represented by the molar decadic extinction coefficient

$$
\varepsilon_{p}(v)=\frac{8 \pi^{3} N_{\mathrm{A}}}{3000 h c \ln (10)} v_{p}\left\langle 1_{p}\left|Q_{p}\right| 0_{p}\right\rangle^{2} D_{p} f\left(v_{p}, v\right)
$$

Vibrational circular dichroism intensity is given as the difference of the extinction coefficients for left and right circularly polarized light

$$
\Delta \varepsilon_{p}(v)=\frac{32 \pi^{3} N_{\mathrm{A}}}{3000 h c \ln (10)} v_{p} \operatorname{Im}\left\{\left\langle 1_{p}\left|Q_{p}\right| 0_{p}\right\rangle\left\langle 1_{p}\left|P_{p}\right| 0_{p}\right\rangle\right\} R_{p} f\left(v_{p}, v\right) .
$$

$N_{\mathrm{A}}$ is Avogadro's number, and $v_{p}$ the vibrational frequency of mode vibrational $p . f$ is the function, which describes the band shape while $D_{p}$ and $R_{p}$ are respectively called the reduced dipole strength and the reduced rotational strength obtained by double contraction of second rank tensors describing respectively the dipole and rotational strength. ${ }^{4}$ In pyVib, these quantities are decomposed according to eq. (5).

The situtation for Raman and especially ROA is far more complicated as one has the possibility to measure them with different scattering geometries. The three polarization schemes by which ROA scattering can be mesured, are called incident circular polarization (ICP), scattered circular polarization (SCP), and dual circular polarization (DCP). In ICP, the incident light is modulated between right and left polarizations while the scattered light is analyzed with respect to either one of the polarization (perpendicular and parallel) or natural polarization. In SCP, the incident light is either linearly or naturally polarized while the circular content of the scattered light is measured. In $\mathrm{DCP}_{I}$, one of the two variants of DCP, the circularities of the incident light and of the scattered light are of the same kind. $\mathrm{DCP}_{I I}$ differs from $\mathrm{DCP}_{I}$, in that the circularities of incident and scattered lights are opposite. However, the differential Raman scattering cross sections can be described by the general formulae, regardless of the experimental configurations

$$
d \sigma_{p}=K_{p}\left\langle 1_{p}\left|Q_{p}\right| 0_{p}\right\rangle^{2}\left[C_{1} a_{p}^{2}+C_{2} \beta_{p}^{2}\right] d \Omega
$$

with $d \Omega$ being the infinitesimally small element of the solid angle. For instance, $C_{1}=90$ and $C_{2}=14$ for forward and backward scattering SCP.

For ROA, the differential scattering cross sections take the form

$$
-\Delta d \sigma_{p}=\frac{4 K_{p}}{c}\left\langle 1_{p}\left|Q_{p}\right| 0_{p}\right\rangle^{2}\left[C_{3} a G_{p}+C_{4} \beta_{G p}^{2}+C_{5} \beta_{A p}^{2}\right] d \Omega
$$

Table 1. Linear Combinations of Invariants Corresponding to Theoretical Differential and Integral Cross Sections for the Experimental Methods Implemented in pyVib Program.

\begin{tabular}{llcccrr}
\hline Scatt. angle $\left(^{\circ}\right)$ & Configuration & $C_{1}$ & $C_{2}$ & $C_{3}$ & $C_{4}$ & $C_{5}$ \\
\hline 0 & SCP unpolarized & 90 & 14 & 90 & 2 & -2 \\
180 & SCP unpolarized & 90 & 14 & & 12 & 4 \\
90 & ICP polarized & 90 & 14 & 90 & 14 & 2 \\
& ICP depolarized & & 24 & & 12 & -4 \\
Integral & SCP & $180 \times 4 \pi / 3$ & $40 \times 4 \pi / 3$ & $180 \times 2 \pi / 3$ & $40 \times 2 \pi / 3$ & \\
Integral & ICP & $180 \times 8 \pi / 3$ & $40 \times 8 \pi / 3$ & $180 \times 4 \pi / 3$ & $40 \times 4 \pi / 3$ & \\
\hline
\end{tabular}

Coefficients $C_{i}$ are used in eqs. (19) and (20). 
Table 2. Parameters of the six Gaussian used to approximate the Lorentz band shape.

\begin{tabular}{|c|c|c|c|c|c|c|}
\hline & 1 & 2 & 3 & 4 & 5 & 6 \\
\hline$c_{i}$ & $2.41 \times 10^{-1}$ & $4.32 \times 10^{-1}$ & $2.34 \times 10^{-1}$ & $7.37 \times 10^{-2}$ & $1.60 \times 10^{-2}$ & $2.1 \times 10^{-3}$ \\
\hline$a_{i}$ & $2.26 \times 10^{-1}$ & $4.04 \times 10^{-1}$ & $7.69 \times 10^{-1}$ & $1.61 \times 10^{+0}$ & $3.97 \times 10^{+0}$ & $1.38 \times 10^{+1}$ \\
\hline
\end{tabular}

Parameters $a_{i}$ : standard deviations for each of the six Gauss curves.

Parameters $c_{i}$ : coefficients of each Gauss function in the linear combination.

where $K_{p}=10^{7} \pi^{2} \mu_{0}^{2} c^{4} v_{p}^{3} \nu_{0}$ is a constant, which depends on the frequency $v_{0}$ of the exciting light and the frequency $v_{p}$ of the scattered light, while $\mu_{0}$ is the permeability of the vacuum. The minus sign is due to the sign convention in ROA, which is opposite to the one used in optical activity in general. ${ }^{3,10,11}$ Table 1 shows the values of coefficients $C_{i}$ for most used experimental configurations. In addition to the differential cross sections, Table 1 presents the coefficients for integral SCP and ICP as also implemented in pyVib. These coefficients are obtained by double integration of eqs. 19 and 20 over $\Omega$. In pyVib, these theoretical differential and integral cross sections are multiplied by the Boltzman factor

$$
\frac{1}{1-\exp \left(-100 h c \Delta v_{p} / k T\right)}
$$

with $k$ being the Boltzman's constant and $h$ the Planck's constant. The temperature is fixed at $293^{\circ} \mathrm{K}$ in pyVib as almost all Raman/ROA spectra are measured at room temperature. Then, convolution of the discrete intensities and frequencies are cross sections per steradian and per $\mathrm{cm}^{-1}$ (differential) and cross sections per $\mathrm{cm}^{-1}$ (integral).

\section{Convolution}

Discrete frequencies and intensities calculated by pyVib from Gaussian $f c h k$ outputs are then convoluted to produce realistic spectra. The voigt profile one expects for IR/VCD and Raman/ROA bands is calculated according to an elegant and efficient approach, which exploits the advantage that a convolution of two gaussian functions is still a gaussian function. In this approach, the Lorentz function is approximated by a linear combination of six Gaussian functions whose coefficients are available in the literature. ${ }^{4}$ Finally, the shape function for voigt profile, which is the convolution of the approximate Lorentz function with a Gaussian line shape, takes the form:

$$
V(x)=\frac{2}{\pi} \sum_{i=1}^{6}\left(4 k^{2}+\left(\frac{a_{2}^{2}}{a_{i}^{2}}\right)\right)^{-1 / 2} \exp \left(\frac{-x^{2}}{2\left[\left(2 k a_{i}^{2}\right)+a_{2}^{2}\right]}\right)
$$

where $2 k$ is the FWHM of the approximated Lorentz function and $a_{i}$ and $c_{i}$ are the fitted coefficients and exponents for the six Gaussian functions (see Table 2).

\section{Summary}

In conclusion, the main features of pyVib program are noted in brief. The program processes $f c h k$ ascii output files from GAUSSIAN applications, reading the Hessian, the gradients of polarizabilities $\alpha, G^{\prime}$, and $A$, as well as APTs and AATs. Then, vibrational analysis is performed and cross sections are calculated. Additionally, one can visualize GCMs and ACPs as 2D and 3D representations, respectively. It can also write text output files allowing one to visualize computed intensities with other programs.

\section{Acknowledgments}

The use of Python was suggested by Prof. Werner Hug. I am grateful for helpful discussions with him and Mr. Jacques Haesler.

\section{References}

1. Gaussian03, Gaussian Inc., Pittsburgh, PA.

2. Hug, W. Chem Phys 2001, 264, 53.

3. Buckingham, A. Faraday Discuss 1994, 99, 1.

4. Hug, W.; Haesler, J. Int J Quantum Chem 2005, 104, 695.

5. Wilson, E.; Decius, J.; Cross, P. Molecular Vibrations: The Theory of Infrared and Raman Vibrational Spectra; McGraw Hill: New York, 1955.

6. Hug, W.; Zuber, G.; de Meijere, A.; Khlebinkov, A. F.; Hansen, H. Helvetica Chim Acta 2001, 84, 1.

7. Hug, W.; Chalmers, J. M.; Griffith, P. R. Eds. Handbook of Vibrational Spectroscopy; Wiley: Chichester, 2002; Vol. 1; pp. 745-758.

8. Barron, L. Molecular Light Scattering and Optical Activity, 2nd ed.; Cambridge University Press: Cambridge, 2004.

9. Freedman, T.; Nafie, L.; Evans, M.; Kielich, S. Eds. Wiley: New York, 1994; p. 207, Part 3.

10. Hug, W.; Surbeck, H. Chem Phys Lett 1979, 60, 186.

11. Barron, L. Chem Phys Lett 1983, 102, 285. 